\title{
MicroRNA-497 inhibits tumor growth through targeting insulin receptor substrate 1 in colorectal cancer
}

\author{
YANJIE XU ${ }^{1,2^{*}}$, JIANPING CHEN ${ }^{3 *}$, CAO GAO $^{4^{*}}$, DANXIA ZHU ${ }^{1,2}$, \\ XIAOLI XU ${ }^{2,5}$, CHANGPING WU ${ }^{1,2}$ and JINGTING JIANG ${ }^{2,5}$ \\ ${ }^{1}$ Department of Oncology; ${ }^{2}$ Cancer Immunotherapy Engineering Research Center of Jiangsu; Departments of \\ ${ }^{3}$ Gastroenterology, ${ }^{4}$ Anesthesiology and ${ }^{5}$ Tumor Biological Treatment, The Third Affiliated Hospital of Soochow University, \\ Changzhou, Jiangsu 213003, P.R. China
}

Received June 12, 2015; Accepted February 27, 2017

DOI: $10.3892 / \mathrm{ol} .2017 .7033$

\begin{abstract}
MicroRNAs (miRNAs) have been demonstrated to serve an important role in diverse biological processes and cancer progression. Downregulation of microRNA-497 (miR-497) has been observed in human colorectal cancer (CRC) tissues, but the function of miR-497 in CRC has not been well investigated. In the present study, it was demonstrated that expression of miR-497 was significantly downregulated in human CRC tissues compared to adjacent normal tissues. Enforced expression of miR-497 inhibited proliferation, migration and invasion abilities of CRC cell lines SW1116 and SW480. Furthermore, overexpression of miR-497 inhibited phosphoinositide 3-kinase/AKT and mitogen-activated protein kinase/extracellular signal-regulated kinase signaling by targeting insulin receptor substrate 1 (IRS1). In human clinical specimens, IRS1 was inversely correlated with miR-497 in CRC tissues. Collectively, the results of the present study demonstrate that miR-497 is a tumor suppressor miRNA and indicate its potential application for the treatment of human $\mathrm{CRC}$ in the future.
\end{abstract}

\section{Introduction}

Colorectal cancer (CRC) is the fourth most commonly diagnosed cancer and the third leading cause of cancer-associated mortality in men and women (1). A number of patients are diagnosed in the advanced disease stage, despite efforts

Correspondence to: Professor Changping Wu, Department of Oncology, The Third Affiliated Hospital of Soochow University, 185 Juqian Street, Changzhou, Jiangsu 213003, P.R. China

E-mail:wcpzlk@163.com

Professor Jingting Jiang, Department of Tumor Biological Treatment, The Third Affiliated Hospital of Soochow University, 185 Juqian Street, Changzhou, Jiangsu 213003, P.R. China

E-mail: jiangjingting@suda.edu.cn

${ }^{*}$ Contributed equally

Key words: microRNA, microRNA-497, tumor growth, insulin receptor substrate 1 , colorectal cancer and improvements in early diagnosis (2). Although a variety of therapeutic options are available for CRC patients, including surgery, chemotherapy and radiotherapy, the five-year survival rate of CRC has not significantly improved (3). Previous studies have demonstrated that genetic and epigenetic alterations are involved in the tumorigenesis of $\mathrm{CRC}$, including the activation of oncogenes and/or the suppression of tumor suppressor genes. Increasing evidence suggests that microRNAs (miRNAs/ miRs) may serve key roles in the development of CRC (4-6).

miRNAs, a class of endogenous single-stranded non-coding RNAs, have been associated with various types of cancer (7). miRNAs serve an essential role in the regulation of gene expression and are involved in numerous important physiological and pathological processes, including development, differentiation and tumorigenesis (8-10). By binding the 3'-untranslated region (3'-UTR) of mRNA, miRNA suppresses protein synthesis through mRNA degradation or translational repression. As a result, miRNAs may act as either tumor suppressors or oncogenes (11-13). It is becoming increasingly evident that miRNAs serve important roles in cancer etiology. As a tumor suppressor gene in several cancer types, miR-497 is able to affect tumor cell growth, migration, invasion and apoptosis $(14,15)$. To date, certain genes have been identified as miR-497 targets, including Nrdp1, Cyclin E1, B-cell lymphoma 2 and insulin-like growth factor 1 receptor (IGF1R) (14,16-18). However, the role and underlying mechanism of miR-497 in regulating tumorigenesis remains to be further elucidated. Notably, it has been reported that miR-497 regulates malignant behavior of CRC cells by targeting IGF1R (16). In the present study, it was observed that miR-497 targeted insulin receptor substrate 1 (IRS1), which is characterized as a typical cytosolic adaptor protein in both insulin receptor (IR) and IGF1R signaling. Studies have indicated that nuclear IRS1 is able to participate in modulating the transcriptional activity of genes involved in cell growth and proliferation $(19,20)$. Nuclear IRS1 binds $\beta$-catenin and works as a transcriptional modulator to stimulate cyclin D1 and c-myc promoter activities a number of cancer types, where it acts as an oncogene, including in pancreatic (21) and breast cancer (22). Epidemiological investigation has revealed that IRS1 is important in the etiology of CRC (23). 
It has been reported that miR-497 may serve roles in CRC via affecting various signaling pathways (16). In the present study, the aim was to identify the roles of miR-497 and its molecular and cellular mechanisms in CRC. Ectopic expression of miR-497 inhibited proliferation, migration and invasion of CRC cells by suppressing a key target, IRS1. Furthermore, the present study defined the molecular mechanism of the tumor suppressive function of miR-497 by inhibiting both phosphoinositide 3-kinase (PI3K)/AKT and mitogen-activated protein kinase (MAPK)/extracellular signal-regulated kinase (ERK) signaling pathways via IRS1 suppression. The results of the present study revealed that miR-497 expression was significantly downregulated in human CRC tissues compared with adjacent paired normal controls. IRS1 expression in CRC tumors was negatively correlated with miR-497 expression. The results of the present study revealed a novel mechanism of miR-497 in CRC, and demonstrate its potential to be used as a novel strategy to develop miR-497-based therapeutics.

\section{Materials and methods}

Cell culture and clinical tissues. Human CRC cell lines SW1116 and SW480 (purchased from Nanjing KeyGen Biotech Co., Ltd., Nanjing, China) were cultured in RPMI-1640 medium (Gibco; Thermo Fisher Scientific, Inc., Waltham, MA, USA), and HEK-293T cells (American Type Culture Collection, Manassas, VA, USA) were cultured in Dulbecco's modified Eagle's medium (Gibco; Thermo Fisher Scientific, Inc.) supplemented with $10 \%$ fetal bovine serum (FBS) (Gibco; Thermo Fisher Scientific, Inc.), 100 IU/ml penicillin and $100 \mathrm{mg} / \mathrm{ml}$ streptomycin. All cells were incubated at $37^{\circ} \mathrm{C}$ in an atmosphere of $5 \% \mathrm{CO}_{2}$.

Colon cancer tissues and adjacent normal tissues were collected from clinical patients undergoing colon cancer resection. All tissue samples were immediately snap-frozen in liquid nitrogen following surgery. All human CRC samples were divided into Grade I, Grade II and Grade III-IV according to the WHO classification (24). In total, 50 pairs of CRC tissues and adjacent normal tissues from patients who underwent surgical operations at The Third Affiliated Hospital of Soochow University (Changzhou, China) from August 1, 2013 to July 31,2014 , were obtained for the study. Written informed consent was obtained from all patients. The present study was approved by the review board and ethics committee of The Third Affiliated Hospital of Soochow University.

Lentiviral packaging and stable cell line establishment. The Lentiviral Packaging kit was used (Thermo Fisher Scientific, Inc.) for stably overexpressing miR-497 in CRC cells. Lentivirus carrying miR-497 or negative control (miR-NC) was packaged following according to the manufacturer's protocol. Lentivirus was packaged in HEK-293T cells and secreted into the medium. Cells were transfected with lentivirus carrying miR-497 or miR-NC in the presence of polybrene (Sigma-Aldrich; Merck KGaA, Darmstadt, Germany) and selected by puromycin (Sigma-Aldrich; Merck $\mathrm{KGaA}$ ) for 2 weeks to obtain stable cell lines.

miRNA mimic transfection. Cells were seeded into $6,12,24$, or 96-well plates and incubated at $37^{\circ} \mathrm{C}$ and $5 \% \mathrm{CO}_{2}$ overnight.
miR-497 mimics and miR-NC were chemically synthesized by Shanghai GenePharma Co., Ltd. (Shanghai, China). Cells at $50-70 \%$ confluence were transfected with miR-497 or miR-NC using Lipofectamine 2000 (Invitrogen; Thermo Fisher Scientific, Inc.) according to the manufacturer's protocol. Transfected cells were harvested at 24 or $48 \mathrm{~h}$ following transfection.

RNA extraction and reverse transcription-quantitative polymerase chain reaction ( $R T-q P C R)$. Total RNA was extracted from cultured cells using $1.0 \mathrm{ml}$ of Trizol reagent (Invitrogen; Thermo Fisher Scientific, Inc.) according to the manufacturer's protocol, and purified RNA was stored at $-80^{\circ} \mathrm{C}$ prior to further analysis. RT-qPCR analysis for mature miR-497 was performed in triplicate using the PrimeScript RT Reagent kit (Takara Biotechnology Co., Ltd, Dalian, China) according to the manufacturer's protocol. Briefly, $500 \mathrm{ng}$ total RNA was reverse transcribed into cDNA, and qPCR was performed using SYBR Premix DimerEraser (Takara Biotechnology Co., Ltd.) on a 7900HT system. The thermocycling conditions were as follows: Pre-denaturation at $95^{\circ} \mathrm{C}$ for $30 \mathrm{sec}$, followed by 40 cycles of $95^{\circ} \mathrm{C}$ for $5 \mathrm{sec}, 55^{\circ} \mathrm{C}$ for $30 \mathrm{sec}$ and $72^{\circ} \mathrm{C}$ for $31 \mathrm{sec}$. The sequences of the primers used for RT-qPCR were as follows: miR-497 RT, 5'-CTCAACTGGTGTCGTGGA GTCGGCAATTCAGTTGAGAACA-3'; miR-497-forward (F), 5'-ACACTCCAGCTGGGCAGCAGCACACTGTGG-3'; miR-497-reverse (R), 5'-TGGTGTCGTGGAGTCG-3'; U6 RT, 5'-AACGCTTCACGAATTTGCGT-3'; U6-F, 5'-CTC GCTTCGGCAGCACA-3'; and U6-R, 5'-TGGTGTCGTGGA GTCG-3'. The miR-497 expression in each group was determined relative to that of U6, and fold changes were calculated by relative quantification $\left(2^{-\Delta \Delta \mathrm{Cq}}\right)(25)$.

Cell proliferation assay. Cell Counting Kit-8 (CCK-8; Dojindo Molecular Technologies, Inc., Kumamoto, Japan) assay was used to determine cell viability. Cells were seeded at a density of 2,000 cells per well in 96-well plates and cultured as described above for $48 \mathrm{~h}$ following transfection. After 24 , 48, 72 and $96 \mathrm{~h}$ incubation, CCK-8 was added into each well, followed by an additional $2 \mathrm{~h}$ incubation at $37^{\circ} \mathrm{C}$. Absorbance at a wavelength of $450 \mathrm{~nm}$ was subsequently determined. Experiments were performed in triplicate.

Wound healing assay. Cells were transfected with miR-497 or miR-NC according to the manufacturer's protocol, and subsequently cultured to $95 \%$ confluence in 6-well plates. Cell monolayers were scratched using a $20 \mu 1$ tip to form wound gaps and washed twice with PBS to remove the detached cells. After $24 \mathrm{~h}$, the wound healing was photographed at various time points. The cell migration distances were measured in three different areas to indicate the migration ability of various cell treatments.

Invasion assay. The effect of miR-497 on tumor invasion was investigated using 24-well BD Matrigel invasion chambers (BD Biosciences, Franklin Lakes, NJ, USA) according to the manufacturer's protocol. The transfected cells $\left(5 \times 10^{4}\right)$ were seeded in the upper well of the invasion chamber containing serum-free RPMI-1640, and RPMI-1640 containing 10\% FBS was applied to the lower chamber. After $24 \mathrm{~h}$, any non-invading 
cells on the top well were removed with a cotton swab, while cells in the bottom well were fixed with $3 \%$ paraformaldehyde and stained with $0.1 \%$ crystal violet. Images were captured in three independent fields (magnification, x10). The membrane was air-dried, soaked with $33 \%$ acetic acid (200 $\mu \mathrm{l} /$ well) at room temperature for $15 \mathrm{~min}$ and subsequently transferred to a 96-well plate. The absorbance at a wavelength of $570 \mathrm{~nm}$ was recorded (Synergy 2; BioTek Instruments, Inc., Winooski, VT, USA). Results were obtained from three independent experiments.

Western blotting. Cells were treated as previously described, and were harvested after $24 \mathrm{~h}$ and lyzed in radioimmunoprecipitation assay buffer supplemented with protease inhibitors (100 mM Tris-HCl, pH 7.4, 150 mM NaCl, 1\% Triton X-100, $5 \mathrm{mM}$ EDTA, $2 \mathrm{mM}$ phenylmethylsulfonyl fluoride, $1 \%$ deoxycholate acid, $0.1 \%$ SDS, 2 mM DTT, $1 \mathrm{mM}$ sodium orthovanadate, $2 \mathrm{mM}$ leupeptin and $2 \mathrm{mM}$ pepstatin) on ice for $30 \mathrm{~min}$ (26). Following centrifugation, protein concentrations were determined by the bicinchoninic acid method (Beyotime Institute of Biotechnology, Haimen, China), and $20 \mu \mathrm{g}$ protein was then separated by $10 \%$ SDS-PAGE. Subsequently, protein was electrically transferred onto a nitrocellulose membrane (Whatman GmbH, Dassel, Germany). The membrane was incubated with anti-IRS1 (1:1,000; catalog no. CST 2382; Cell Signaling Technology, Inc., Danvers, MA, USA) and anti-GAPDH (1:5,000; catalog no. MB001; Bioworld Technology, Inc., St. Louis Park, MN, USA) antibodies at $4^{\circ} \mathrm{C}$ overnight, followed by incubation at room temperature for $2 \mathrm{~h}$ with a secondary antibody (catalog no. 31460; Thermo Fisher Scientific, Inc.) diluted 1:2,000 for IRS1 detection and 1:5,000 for GAPDH detection. Antibodies against phosphorylated (p)-AKT (Ser473) (1:1,000; catalog no. CST 4060), AKT (1:2,000; catalog no. CST 9272), p-ERK1/2 (1:1,000; catalog no. CST 14474) and ERK1/2 (1:2,000; catalog no. CST 4348) were purchased from Cell Signaling Technology, Inc., and were incubated at $4{ }^{\circ} \mathrm{C}$ overnight, followed by incubation at room temperature for $2 \mathrm{~h}$ with the aforementioned secondary antibody a 1:2,000 dilution. ECL Detection System (Thermo Fisher Scientific, Inc.) was used for protein signal detection. The density of the signals was quantified using Image J software with the ChemiDoc Imaging System (Bio-Rad Laboratories, Inc., Hercules, CA, USA). GAPDH was used as a control for normalization.

Luciferase reporter assay. Prediction of miR-497 binding sites was performed using TargetScan software using the key words 'IRS1' and 'human species'. TargetScan (www. targetscan.org) and miRanda (www.microrna.org) predict biological targets of miRNAs by searching for the presence of conserved $8 \mathrm{mer}, 7 \mathrm{mer}$ and $6 \mathrm{mer}$ sites that match the seed region of each miRNA (27). A fragment of 3'-UTR of IRS1 containing the putative miR-497 binding site was amplified by PCR. To generate a construct containing the mutant miR-497 binding site, two nucleotides corresponding to the 5'-seeding region of the miR-497 binding site on the wild type fragment were substituted. Its complementary sequence in the 3'-UTR of IRS1 (UGCUGCU) was replaced by UCCACCA. The PCR products were digested using SacI and HindIII, inserted into pMIR-REPORTER (Promega Corporation, Madison, WI,
USA) and validated by DNA sequencing. Constructs were transfected into HEK-293 cells in 24-well plates and co-transfected with miR-497 or miR-NC. Luciferase assays were performed $24 \mathrm{~h}$ post-transfection using the Dual Luciferase Reporter Assay system (Promega Corporation).

Xenograft studies. For tumor growth assay, male nude mice [BALB/cA-nu (nu/nu), 6-week-old, weighting 20-25 g] were purchased from Shanghai Laboratory Animal Center (Chinese Academy of Sciences, Shanghai, China), and animals were maintained under special pathogen-free (SPF) conditions. Mice were housed at a temperature of $26-28^{\circ} \mathrm{C}$, relative humidity of $40-60 \%$ and $10 \mathrm{~h}$ light $/ 14 \mathrm{~h}$ dark cycle, with access to food and water ad libitum. Animal protocols were approved by the Animal Welfare Committee of Soochow University (The Third Affiliated Hospital of Soochow University). Aliquots of cells $\left(5 \times 10^{6}\right)$ were suspended in $150 \mu \mathrm{l}$ of FBS-free RPMI-1640 medium and subcutaneously injected into each side of the posterior flank of nude mice. Tumor size was measured using vernier calipers every 2 days when they became visible, and the tumor volume was calculated according to the formula: Volume $=0.5 \mathrm{x}$ length $\mathrm{x}$ width ${ }^{2}$. Mice were sacrificed on day 22 following injection of tumor cells, and xenografts were collected. The animals were euthanized by cervical dislocation (28).

Statistical analysis. All experiments were performed in triplicate, and data were analyzed with GraphPad Prism 5 (GraphPad Software, Inc., La Jolla, CA, USA). The correlation between the expression of miR-497 and IRS1 in CRC tissues was analyzed using Spearman's rank test. Statistical evaluation for data analysis was determined by the Student's t-test. $\mathrm{P}<0.05$ was considered to indicate a statistically significant difference.

\section{Results}

Expression of miR-497 is significantly downregulated in CRC tissue. To identify the role of miR-497 in the development of colorectal cancer, the expression level of miR-497 was analyzed in 50 pairs of CRC tissues and adjacent normal tissues. RT-qPCR analysis revealed that the miR-497 level was significantly downregulated in CRC tissues (Fig. 1A). It was also observed that reduced levels of miR-497 in CRC patients were positively correlated with the status of pathology classification (Fig. 1B). These results indicated that the progressive loss of miR-497 may be associated with CRC disease progression.

miR-497 suppresses CRC cell growth, migration and invasion in vitro. To investigate the direct role of miR-497 in CRC cells, the present study established stable cell lines by transfecting SW1116 and SW480 cells with miR-497 overexpressing lentiviral vector (Lv-miR-497) or a control lentiviral empty vector ( $\mathrm{Lv}$-miR-NC), followed by puromycin selection. Subsequently, CRC cell proliferation was detected in vitro. Cell viability assay indicated that overexpression of miR-497 significantly reduced the cell proliferation rate at $48 \mathrm{~h}$ following cell seeding in SW1116 and SW480 cells, 
A

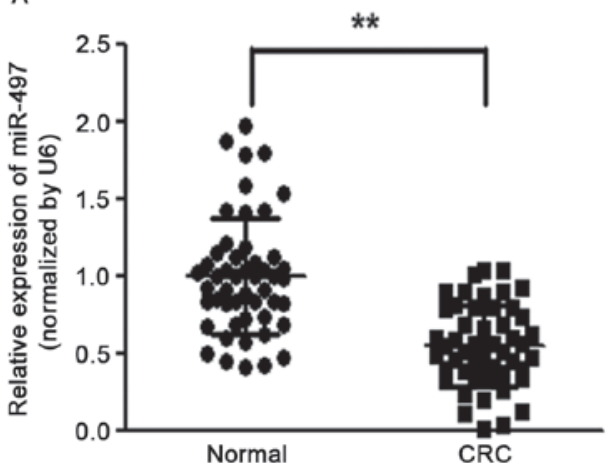

$B$

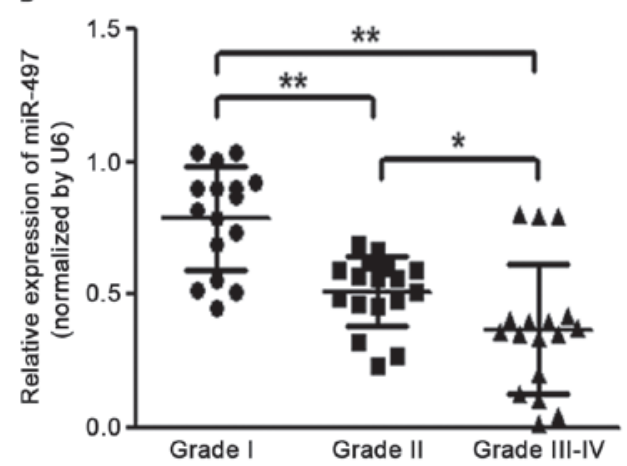

Figure 1. miR-497 is significantly downregulated in CRC tissue. (A) Relative miR-497 expression levels were analyzed by reverse transcription-quantitative polymerase chain reaction in 50 pairs of human CRC tissues and adjacent normal tissues. U6 RNA level was used as an internal control. (B) miR-497 expression in three different grades of CRC samples. According to the pathological classification, the 50 pairs of human CRC tissues were divided into three groups: World Health Organization Grade I, Grade II and Grade III-IV. Data represent the mean \pm standard deviation of three replicates. "Indicates significant difference at $\mathrm{P}<0.05 ;{ }^{* *}$ indicates significant difference at $\mathrm{P}<0.01$. miR, microRNA; CRC, colorectal cancer.

compared with the LV-miR-NC group (Fig. 2A). Since invasion and migration are key characteristics of malignant tumors, the present study investigated the effects of miR-497 on invasion and migration in vitro. Forced expression of miR-497 also markedly suppressed the invasion of SW1116 and SW480 cells in migration assays, as well as wound healing assays (Fig. 2B and C).

miR-497 inhibits AKT and ERK1/2 signaling pathways via targeting IRSI. To investigate the underlying mechanism of miR-497 in CRC, the present study analyzed TargetScan and miRanda databases. It was observed that miR-497 likely regulates the IRS1 gene, since the 3'-UTR of IRS1 contained the binding site for the seed region of miR-497. IRS1 is characterized as a typical cytosolic adaptor protein in both IR and IGF1R signaling. According to the putative binding site of miR-497 in the 3'UTR of the IRS1 gene, the present study initially constructed two types of plasmids containing the luciferase reporting gene and wild-type or mutant IRS1 3'UTR and cotransfected a miR-497 mimic into HEK-293T cells; cells co-transfected with a miR-497 mimic and wild-type IRS1 3'UTR demonstrated a significant decrease in luciferase activity. However, in the mutant group, no detectable change in luciferase activity was observed (Fig. 3A), suggesting that miR-497 suppressed the transcription of the IRS1 gene by targeting the putative 3'UTR of IRS1 mRNA independently. Western blotting analysis was conducted to determine IRS1 protein expression. The results revealed that the IRS1 expression in SW116 and SW480 cells transfected with miR-497 mimics was downregulated at the protein level, compared with cells transfected with negative control (Fig. 3B). These data demonstrated that miR-497 directly targeted IRS1 by binding its seed region to the 3'-UTRs in CRC cells. The present study additionally examined the IRS1 expression at the protein level in human CRC specimens and normal tissues. The results demonstrated that the average expression level of IRS1 was significantly increased in tumor tissue compared with normal tissue (Fig. 3C). Further analysis revealed the significant reciprocal association of expression levels of IRS1 with miR-497 in the same human CRC tissue (r=-0.6247; Fig. 3D).
AKT and ERK1/2 signaling pathways act as major downstream regulators of IRS1 signaling, which are critical in mitogenesis and oncogenesis. Cellular levels of p-AKT and p-ERK1/2 were significantly changed in SW1116 and SW480 cells stably expressing miR-497 compared with miR-NC, but the changes in AKT and ERK1/2 were not statistically significant (Fig. 3E). To additionally investigate whether the overexpression of IRS1 affected the expression of p-AKT and p-ERK1/2, cells were co-transfected with or without pCMV6-IRS1 cDNA. The results of the present study demonstrated that forced expression of IRS1 restored miR-497-inhibited cellular levels of p-AKT and p-ERK1/2. These data revealed that miR-497 inhibited AKT and ERK1/2 signaling pathways via targeting IRS1 (Fig. 3E).

miR-497 inhibits tumor growth in vivo. To investigate the effect of miR-497 on tumor growth, SW1116 cells overexpressing miR-497 or miR-NC were subcutaneously injected into the posterior flanks of nude mice $(n=6)$. Xenograft tumor volumes were determined every 2 days after they had become visible. Nude mice were sacrificed on day 22 following injection of tumor cells, and xenografts were collected. Fig. 4A shows representative xenograft tumors. The average tumor weight of the miR-497 overexpression group was markedly reduced by $60 \%$ compared with that of the control (Fig. 4B). On day 16 post-implantation, the tumor growth of the miR 497 overexpression group was significantly reduced compared with that of the control group (Fig. 4C). Total proteins from representative tumor samples were analyzed by western blotting, and the results demonstrated that miR-497 suppressed the expression of IRS1 and p-AKT, as well as p-ERK1/2, in vivo (Fig. 4D). Taken together, these results suggested that miR-497 inhibited tumor growth in vivo via targeting IRS1 and other downstream signaling molecules.

\section{Discussion}

Previous studies have demonstrated that miRNAs serve important roles in carcinogenesis by a number of mechanisms, and certain miRNAs have been reported to be correlated with clinical characteristics and outcomes $(29,30)$. The role of 
A

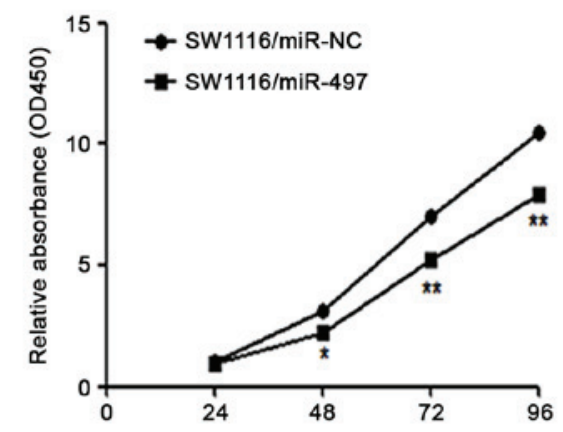

B

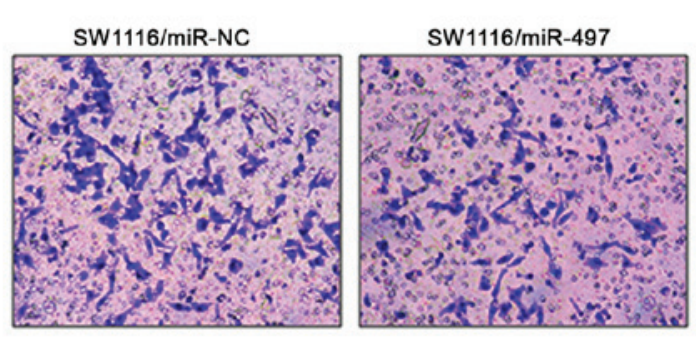

SW480/miR-NC
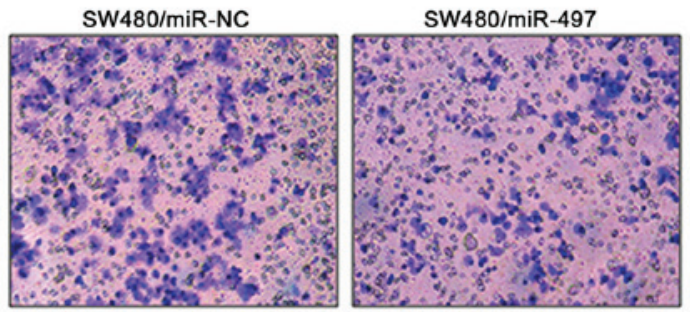
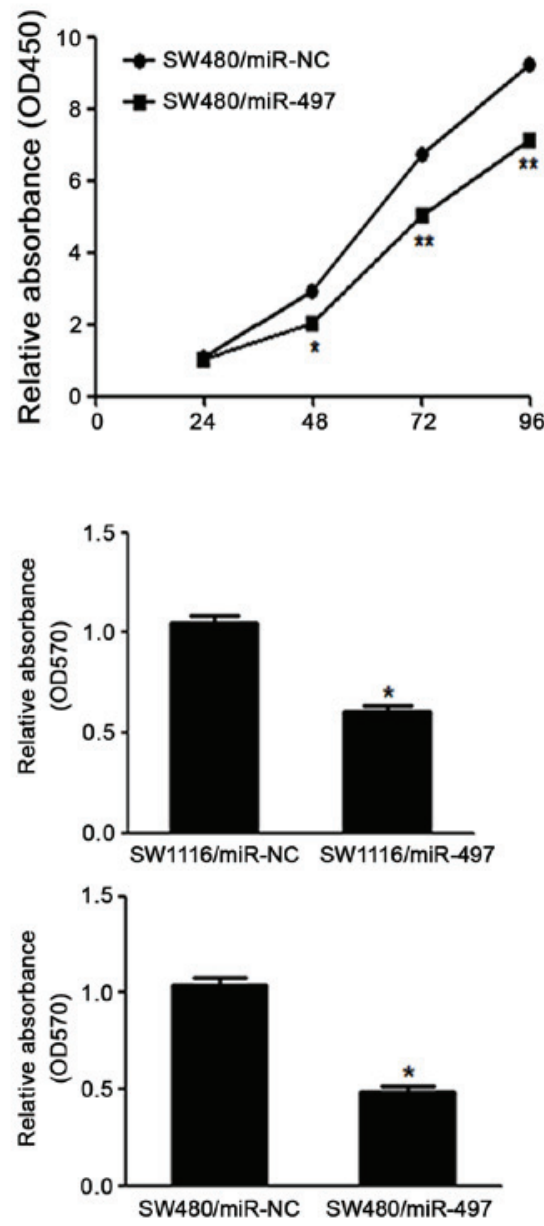

c
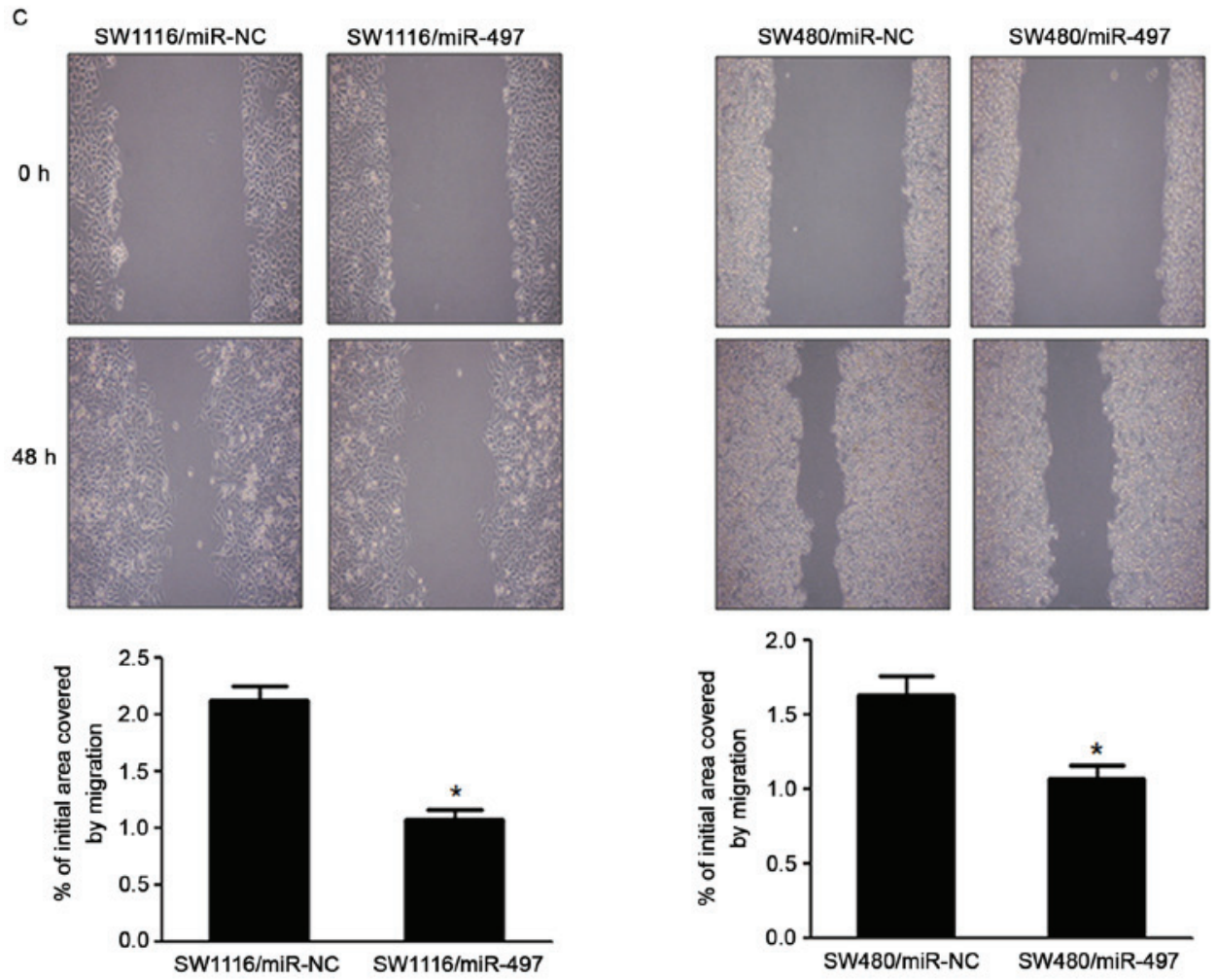

Figure 2. Overexpression of miR-497 inhibits proliferation, invasion and migration in CRC cells. (A) Overexpression of miR-497 arrested cell proliferation in SW1116 and SW480 cells. (B) miR-497 overexpression reduced cell invasion in SW1116 and SW480 cells. (C) Cells were treated as shown. A sterile $20 \mu \mathrm{l}$ pipette tip was used form a wound. The wound gaps were photographed and measured. Forced expression of miR-497 also markedly reduced the wound-healing rate. Data represent the mean \pm standard deviation of three replicates. " Indicates significant difference at $\mathrm{P}<0.05 ;{ }^{* *}$ indicates significant difference at $\mathrm{P}<0.01$. miR, microRNA; OD, optical density; $\mathrm{NC}$, negative control. 
A
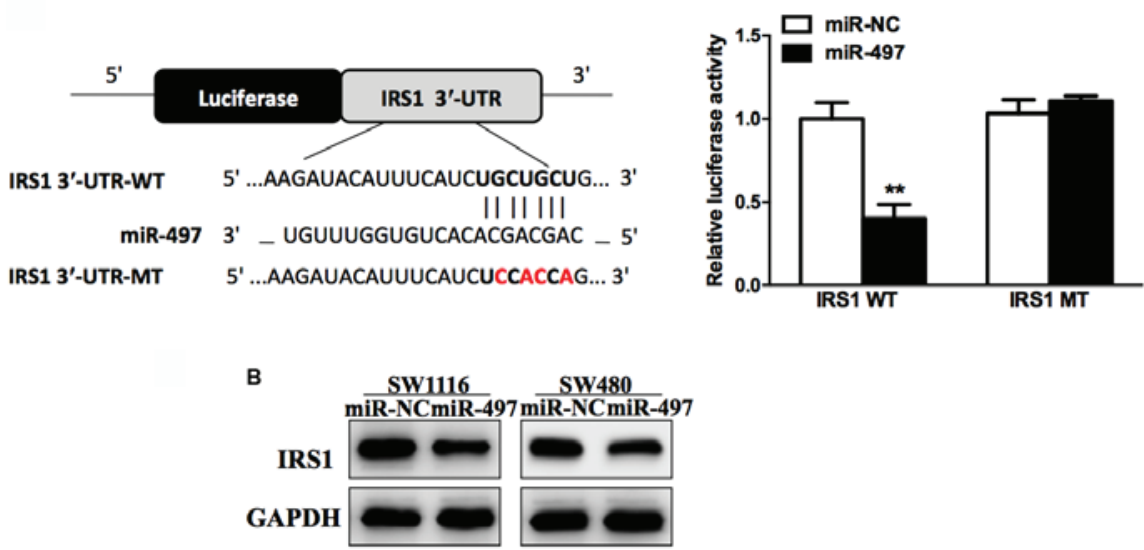

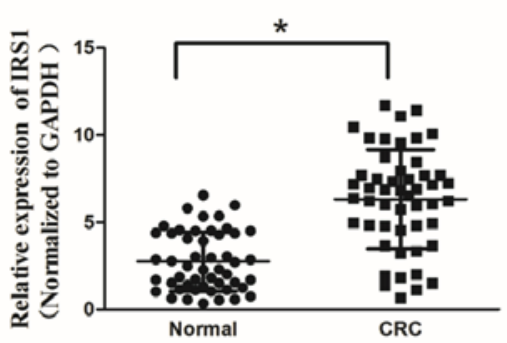

E

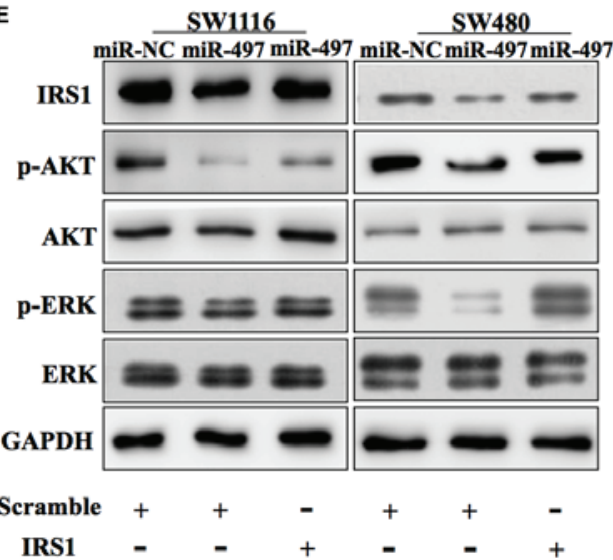

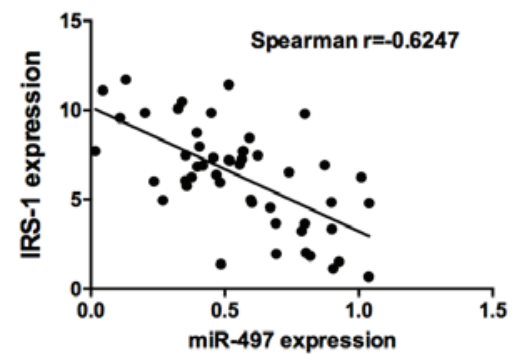
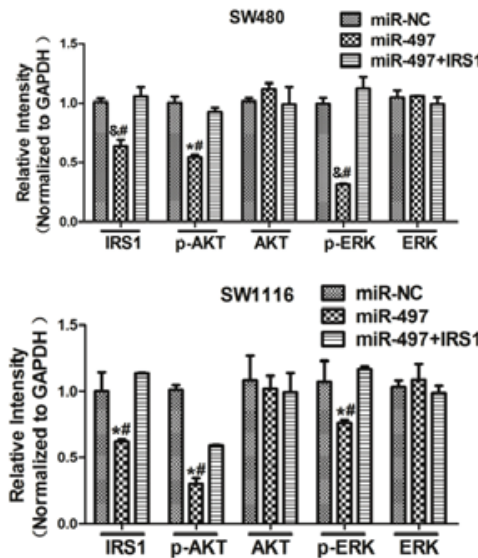

Figure 3. miR-497 inhibits AKT and ERK1/2 signaling pathways via targeting IRS1. (A) Sequence of the miR-497 binding site within the human IRS1 3'-UTR and a schematic diagram of the reporter construct showing the entire IRS1 3'-UTR sequence and the mutant IRS1 3'-UTR sequence. The mutant nucleotides of the IRS1 3'-UTR are labeled in red. Luciferase assay on SW1116 cells, which were co-transfected with miR-NC or miR-497 and a luciferase reporter containing the full length of IRS1 3'-UTR (WT) or a mutant (MT) harboring four mutant nucleotides of the miR-497 binding site. Luciferase activities were measured $24 \mathrm{~h}$ post-transfection. miR-497 markedly suppressed luciferase activity in IRS1 3'-UTR (WT) reporter constructs. The data respresent the mean \pm standard error for separate transfections $(n=4)$. (B) Immunoblotting revealed that expression levels of IRS1 were decreased in cells with miR-497 overexpression. (C) Expression of IRS1 in adjacent normal tissues and human CRC specimens was determined by western blot analysis, and fold changes were obtained from the ratio of IRS1 to GAPDH levels. (D) Spearman's correlation analysis was used to determine the correlation between the expression levels of IRS1 and miR-497 in human CRC specimens. (E) Expression levels of p-AKT and p-ERK1/2 were decreased in cells with miR-497 overexpression, while AKT and ERK1/2 protein levels remained unchanged. Overexpression of IRS1 restored miR-497-inhibited cellular protein levels of p-AKT and p-ERK1/2. Data represent the mean \pm standard deviation of three replicates. ${ }^{*} \mathrm{P}<0.01$ vs. control, ${ }^{* *} \mathrm{P}<0.01,{ }^{*} \mathrm{P}<0.01$ vs. miR- $497+\mathrm{IRS} 1,{ }^{\&} \mathrm{P}<0.05$ vs. control. miR, microRNA; ERK, extracellular signal-regulated kinase; IRS1, insulin receptor substrate 1; UTR, untranslated region; NC, negative control; CRC, colorectal cancer; p, phosphorylated.

certain miRNAs in CRC has also been reported. For example, miR-378 is frequently downregulated in CRC and colorectal cell lines, and upregulation of miR-378 inhibits cell growth and enhances oxaliplatin-induced apoptosis in human CRC (31). miR-194 functions as a tumor suppressor in colorectal carcinogenesis via targeting phosphoinositide-dependent kinase-1/AKT2/X-linked inhibitor of apoptosis protein signaling pathway (32). Previous studies have demonstrated that miR-497 is downregulated in several cancer types, including CRC. Han et al (33) confirmed that miR-497 suppresses the proliferation of human cervical carcinoma HeLa cells by targeting cyclin E1. Another study demonstrated that miR-497 targeted insulin-like growth factor 1 receptor and inhibited proliferation and invasive behavior in colon cancer cells (16). 
A

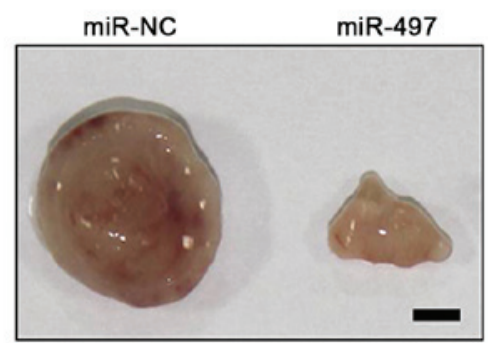

C

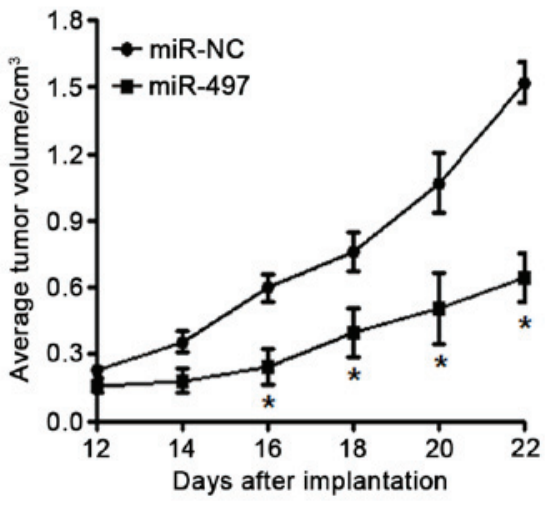

B

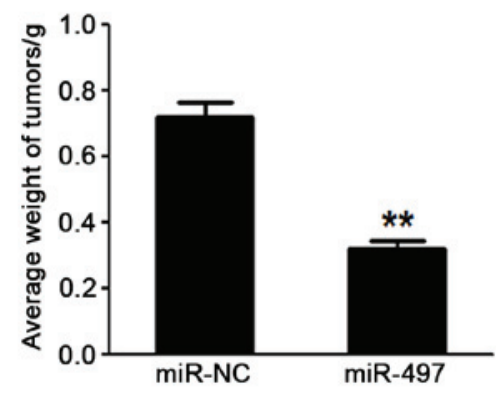

D

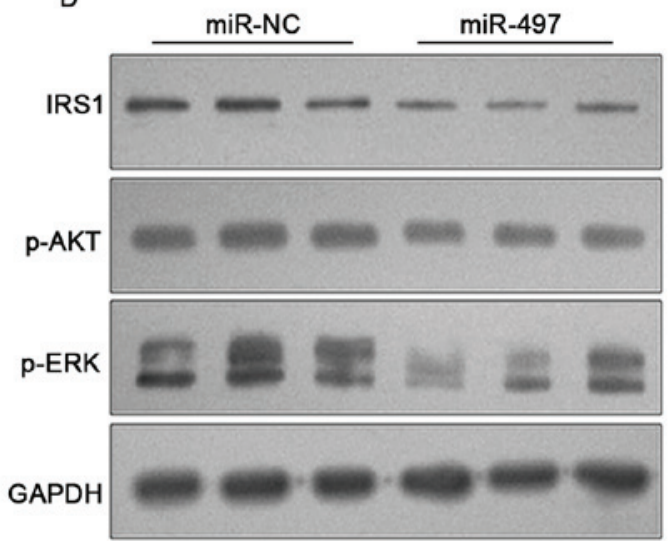

Figure 4. miR-497 inhibits tumor growth in vivo. (A-C) Effect of miR-497 on the growth of SW1116 cells inoculated into nude mice. Male BALB/cA nude mice were subcutaneously injected with $5 \times 10^{6} \mathrm{SW} 1116$ cells infected with lentiviruses harboring miR-NC or miR-497. Tumor volume and weight were monitored over time as indicated, and the tumor was excised and weighed after 22 days. $\mathrm{miR}-497$ caused a decrease in tumor volume and weight. Scale bar $=1$ mm. (D) Expression levels of downstream pathway proteins from the tumor tissues of miR-497 expressing group were reduced compared with those of the miR-NC group. Data represent the mean \pm standard deviation. ${ }^{*}$ Indicates significant difference at $\mathrm{P}<0.05{ }^{*}{ }^{* *}$ indicates significant difference at $\mathrm{P}<0.01$. miR, microRNA; $\mathrm{nC}$, negative control; IRS1, insulin receptor substrate 1; p, phosphorylated; ERK, extracellular signal-regulated kinase.

Consistent with previous studies, the present study identified that miR-497 was downregulated in CRC tissues compared with normal controls, and the degree of miR-497 suppression was negatively correlated with increased grades of human CRC malignancy. Notably, the present study further predicted IRS1 as a target of miR-497 by bioinformatic analysis. For the first time to the best of our knowledge, it was demonstrated that IRS1 was upregulated in CRC tissues and was inversely correlated with miR-497 levels. Thus, in combination with previous research, the present study demonstrated that miR-497 regulated the IGF1R/IRS1 signaling pathway, and may provide novel therapeutic strategies for CRC prevention and treatment.

IRS1 transmits signals from insulin or IGF receptors to activate PI3K/AKT and MAPK pathways, both of which are critical in mitogenesis and oncogenesis $(34,35)$. It has been observed that the expression of IRS1 may promote proliferation in several cell lines (36-38). In the present study, the IRS1 oncogene was experimentally validated as a novel target of miR-497 in vitro and in vivo. Initially, luciferase reporter assay confirmed that miR-497 directly recognized the 3'-UTR of IRS1 transcripts. Furthermore, IRS1 expression was significantly abolished in CRC cells stably expressing miR-497. In addition, a negative correlation was observed between IRS1 protein and miR-497 in clinical samples. Finally, inhibition of IRS1 expression by miR-497 suppressed constitutive phosphorylation of AKT and ERK1/2. These results demonstrate that miR-497 is a tumor suppressor that inhibits the AKT and ERK1/2 signaling pathway through partly targeting IRS1.
In conclusion, the results of the present study provide the first evidence, to the best of our knowledge, that miR-497 serves a significant role in suppressing CRC cell growth via inhibition of IRS1. Although the present study confirmed that miR-497 was able to inhibit the phenotype of CRC by targeting IRS1, there may be other targets of miR-497, which could also affect the growth of CRC cells. However, the present study demonstrated that such an effect was exerted through the suppression of IRS1. Therefore, further studies are required to identify additional targets and signaling pathways of miR-497.

\section{Acknowledgements}

The present study was supported by the National Natural Science Foundation of China (grant nos. 81171653, 30972703, 81201741, 81301960 and 81302047); the Natural Science Foundation of Jiangsu Province (grant no. BK2011246, BK2011247 and BK20130243); The Project of Six Batch of Major Talent Summit (grant no. BRA2010037); Society Development Plans, Department of Science and Technology Changzhou (grant nos. CJ20112020, CZ20110024 and CS20102020) and the Innovative Talents Training Project of Changzhou Health Bureau (grant nos. 2016CZBJ001 and 2016CZLJ022).

\section{References}

1. Jemal A, Siegel R, Ward E, Hao Y, Xu J, Murray T and Thun MJ: Cancer statistics, 2008. CA Cancer J Clin 58: 71-96, 2008. 
2. Siegel R, Desantis C and Jemal A: Colorectal cancer statistics, 2014. CA Cancer J Clin 64: 104-117, 2014.

3. Haggar FA and Boushey RP: Colorectal cancer epidemiology: Incidence, mortality, survival, and risk factors. Clin Colon Rectal Surg 22: 191-197, 2009.

4. Chen J, Chen Y and Chen Z: MiR-125a/b regulates the activation of cancer stem cells in paclitaxel-resistant colon cancer. Cancer Invest 31: 17-23, 2013.

5. Nie J, Liu L, Xing G, Zhang M, Wei R, Guo M, Li X, Xie P, Li L, He F, et al: CKIP-1 acts as a colonic tumor suppressor by repressing oncogenic Smurf1 synthesis and promoting Smurf1 autodegradation. Oncogene 33: 3677-3687, 2014.

6. Faltejskova P, Svoboda M, Srutova K, Mlcochova J, Besse A, Nekvindova J, Radova L, Fabian P, Slaba K, Kiss I, et al: Identification and functional screening of microRNAs highly deregulated in colorectal cancer. J Cell Mol Med 16: 2655-2666, 2012.

7. Hüttenhofer A, Schattner P and Polacek N: Non-coding RNAs: Hope or hype? Trends Genet 21: 289-297, 2005.

8. Ambros V: MicroRNA pathways in flies and worms: Growth, death, fat, stress, and timing. Cell 113: 673-676, 2003.

9. Carlsson J, Davidsson S, Helenius G, Karlsson M, Lubovac Z, Andrén O, Olsson B and Klinga-Levan K: A miRNA expression signature that separates between normal and malignant prostate tissues. Cancer Cell Int 11: 14, 2011.

10. Esquela-Kerscher A and Slack FJ: Oncomirs - microRNAs with a role in cancer. Nat Rev Cancer 6: 259-269, 2006.

11. Caldas C and Brenton JD: Sizing up miRNAs as cancer genes. Nat Med 11: 712-714, 2005

12. Calin GA and Croce CM: MicroRNA signatures in human cancers Nat Rev Cancer 6: 857-866, 2006.

13. Kefas B, Godlewski J, Comeau L, Li Y, AbounaderR, Hawkinson M, Lee J, Fine H, Chiocca EA, Lawler S and Purow B: microRNA-7 inhibits the epidermal growth factor receptor and the Akt pathway and is down-regulated in glioblastoma. Cancer Res 68: 3566-3572, 2008.

14. Luo Q, Li X, Gao Y, Long Y, Chen L, Huang Y and Fang L: MiRNA-497 regulates cell growth and invasion by targeting cyclin E1 in breast cancer. Cancer Cell Int 13: 95, 2013.

15. Zhao WY, Wang Y, An ZJ, Shi CG, Zhu GA, Wang B, Lu MY, Pan CK and Chen P: Downregulation of miR-497 promotes tumor growth and angiogenesis by targeting HDGF in non-small cell lung cancer. Biochem Biophys Res Commu 435: 466-471, 2013.

16. Guo ST, Jiang CC, Wang GP, Li YP, Wang CY, Guo XY, Yang RH, Feng Y, Wang FH, Tseng HY, et al: MicroRNA-497 targets insulin-like growth factor 1 receptor and has a tumour suppressive role in human colorectal cancer. Oncogene 32: 1910-1920, 2013.

17. Zhu W, Zhu D, Lu S, Wang T, Wang J, Jiang B, Shu Y and Liu P: miR-497 modulates multidrug resistance of human cancer cell lines by targeting BCL2. Med Oncol 29: 384-391, 2012.

18. Jiang Y, Meng Q, Qi J, Shen H and Sun S: MiR-497 promotes metastasis of colorectal cancer cells through Nrdp1 inhibition. Tumour Biol 36: 7641-7647, 2015.

19. Chen J, Wu A, Sun H, Drakas R, Garofalo C, Cascio S, Surmacz E and Baserga R: Functional significance of type 1 insulin-like growth factor-mediated nuclear translocation of the insulin receptor substrate-1 and beta-catenin. J Biol Chem 280: 29912-29920, 2005.

20. Wu A, Chen J and Baserga R: Nuclear insulin receptor substrate-1 activates promoters of cell cycle progression genes. Oncogene 27: 397-403, 2008.

21. Bergmann U, Funatomi H, Kornmann M, Beger HG and Korc M: Increased expression of insulin receptor substrate-1 in human pancreatic cancer. Biochem Biophys Res Commun 220: 886-890, 1996.
22. Chang Q, Li Y, White MF, Fletcher JA and Xiao S: Constitutive activation of insulin receptor substrate 1 is a frequent event in human tumors: Therapeutic implications. Cancer Res 62: 6035-6038, 2002.

23. Slattery ML, Samowitz W, Curtin K, Ma KN, Hoffman M, Caan B and Neuhausen S: Associations among IRS1, IRS2, IGF1, and IGFBP3 genetic polymorphisms and colorectal cancer. Cancer Epidemiol Biomarkers Prev 13: 1206-1214, 2004.

24. Fléjou JF: WHO classification of digestive tumors: The fourth edition. Ann Pathol 31: S27-31, 2011 (In French).

25. Livak KJ and Schmittgen TD: Analysis of relative gene expression data using real-time quantitative PCR and the 2(-Delta Delta C(T)) method. Methods 25: 402-408, 2001.

26. Shi ZM, Wang XF, Qian X, Tao T, Wang L, Chen QD, Wang XR, Cao L, Wang YY, Zhang JX, et al: MiRNA-181b suppresses IGF-1R and functions as a tumor suppressor gene in gliomas. RNA 19: 552-560, 2013.

27. Peterson SM, Thompson JA, Ufkin ML, Sathyanarayana $P$, Liaw L and Congdon CB: Common features of microRNA target prediction tools. Front Genet 5: 23, 2014.

28. Shi Z, Chen Q, Li C, Wang L, Qian X, Jiang C, Liu X, Wang X, Li H, Kang C, Jiang T, Liu L, You Y, Liu N and Jiang B: MiR-124 governs glioma growth and angiogenesis and enhances chemosensitivity by targeting R-Ras and N-Ras. Neuro Oncol 16:1341-1353, 2014.

29. Xu J, Wang T, Cao Z, Huang H, Li J, Liu W, Liu S, You L, Zhou L, Zhang T and Zhao Y: MiR-497 downregulation contributes to the malignancy of pancreatic cancer and associates with a poor prognosis. Oncotarget 5: 6983-6993, 2014.

30. Majid S, Dar AA, Saini S, Deng G, Chang I, Greene K, Tanaka Y, Dahiya R and Yamamura S: MicroRNA-23b functions as a tumor suppressor by regulating Zeb1 in bladder cancer. PLoS One 8: e67686, 2013

31. Wang KY, Ma J, Zhang FX, Yu MJ, Xue JS and Zhao JS: MicroRNA-378 inhibits cell growth and enhances L-OHP-induced apoptosis in human colorectal cancer. IUBMB Life 66: 645-654, 2014.

32. Zhao HJ, Ren LL, Wang ZH, Sun TT, Yu YN, Wang YC, Yan TT, Zou W, He J, Zhang Y, et al: MiR-194 deregulation contributes to colorectal carcinogenesis via targeting AKT2 pathway. Theranostics 4: 1193-1208, 2014.

33. Han J, Huo M, Mu M, Liu J and Zhang J: miR-497 suppresses proliferation of human cervical carcinoma HeLa cells by targeting cyclin E1. Xi Bao Yu Fen Zi Mian Yi Xue Za Zhi 30 597-600, 2014 (In Chinese).

34. Scharf JG and Braulke T: The role of the IGF axis in hepatocarcinogenesis. Horm Metab Res 35: 685-693, 2003.

35. Scharf JG, Dombrowski F and Ramadori G: The IGF axis and hepatocarcinogenesis. Mol Pathol 54: 138-144, 2001.

36. Wang LM, Myers MG Jr, Sun XJ, Aaronson SA, White M and Pierce JH: IRS-1: Essential for insulin- and iL-4-stimulated mitogenesis in hematopoietic cells. Science 261: 1591-1594, 1993.

37. Taouis M, Dupont J, Gillet A, Derouet M and Simon J: Insulin receptor substrate 1 antisense expression in an hepatoma cell line reduces cell proliferation and induces overexpression of the Src homology 2 domain and collagen protein (SHC). Mol Cell Endocrinol 137: 177-186, 1998.

38. Waters SB, Yamauchi K and Pessin JE: Functional expression of insulin receptor substrate-1 is required for insulin-stimulated mitogenic signaling. J Biol Chem 268: 22231-22234, 1993. 